\title{
Single Incision Laparoscopic Complete Mesocolic Excision with Central Vascular Ligation for Advanced Transverse Colon Cancer Using Pincer Movement Method
}

\section{Goutaro Katsuno, MD, PhD}

Department of Gastrointestinal and Minimally Invasive Surgery, Mitsuwadai General Hospital, Chiba, Japan.

E-mail: gon9961@gmail.com

Masaki Fukunaga, MD, PhD

Department of Surgery, Juntendo Urayasu Hospital, Juntendo University, Urayasu Japan.

Yasuhiko Nakata, MD, PhD

Department of Gastrointestinal and Minimally Invasive Surgery, Mitsuwadai General Hospital, Chiba, Japan.

Nobuyuki Kubota, MD, PhD

Department of Gastrointestinal and Minimally Invasive Surgery, Mitsuwadai General Hospital, Chiba, Japan.

Teruo Kaiga, MD, PhD

Department of Gastrointestinal and Minimally Invasive Surgery, Mitsuwadai General Hospital, Chiba, Japan.

Takao Mamiya, MD

Department of Gastrointestinal and Minimally Invasive Surgery, Mitsuwadai General Hospital, Chiba, Japan.

\section{Masahiro Yan, MD}

Department of Gastrointestinal and Minimally Invasive Surgery, Mitsuwadai General Hospital, Chiba, Japan.

Naoaki Shimamoto, MD

Department of Gastrointestinal and Minimally Invasive Surgery, Mitsuwadai General Hospital, Chiba, Japan.

(c) Mary Ann Liebert, Inc. DOI: 10.1089/vor.2017.0468
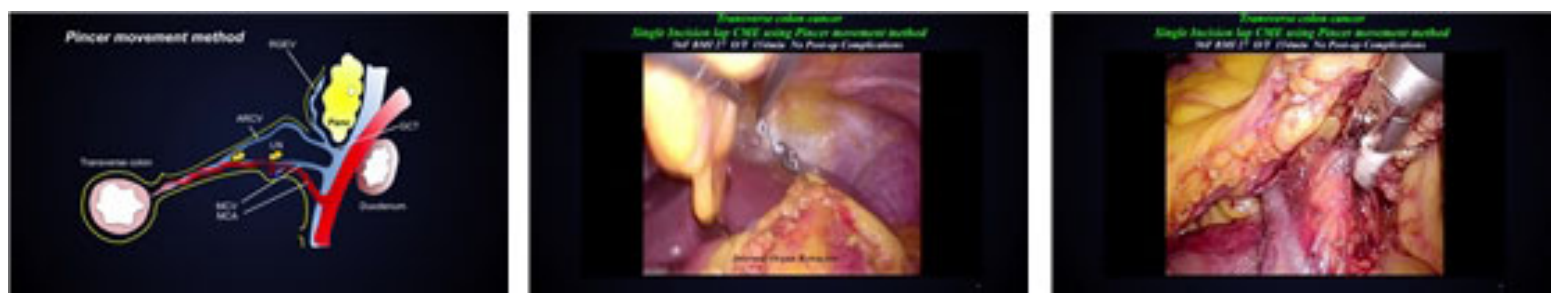

\section{Abstract}

Introduction: Single incision laparoscopic techniques ${ }^{1-3}$ for advanced transverse colon cancer are technically difficult to perform with a complete mesocolic excision (CME) with central vascular ligation (CVL). The main reason for this technical difficulty lies in the limited access to the abdominal cavity and anatomical complexity around the middle colic vessels (MCVs). This video describes a pincer 
movement method using an internal organ retractor to effectively complete the dissection around the MCVs for a tranverse colon cancer.

Methods: The pincer movement method refers to an original strategy used to simplify the anatomical complexity around the MCVs by approaching the transverse mesocolon caudally and cranially. This method approaches the MCVs from both sides of the transverse mesocolon to safely complete the actual circumferential dissection around the base of the MCVs at the superior mesenteric artery (SMA) and superior mesenteric vein (SMV). A $2.5 \mathrm{~cm} \mathrm{Z} \mathrm{type} \mathrm{skin} \mathrm{incision} \mathrm{is} \mathrm{made,} \mathrm{and} \mathrm{an} \mathrm{access} \mathrm{platform} \mathrm{is}$ placed in the small umbilical incision area. The ascending colon is mobilized and the hepatic flexure is dissected using a conventional medial-to-lateral retroperitoneal approach. The omentum is divided, and the divided omentum is retracted toward the cranial space to maintain a working space by using internal organ retractors. The fascia along the inferior border of the pancreas is then transected. As a result, intraoperative pancreatic injury is avoided by securing a free space around the MCVs. Lymph nodes along SMA/SMV can be safely dissected caudally and cranially. The single incision CME with $\mathrm{CVL}$ can be completed along with an extracorporeal anastomosis.

Results: Single incision CME for advanced transverse colon cancer was performed for nine patients. ${ }^{4-6}$ There were four men and five women. The mean age of the patients was $66.5 \pm 6.9$ years (range 58-79) and the mean body mass index was $22.7 \pm 3.8 \mathrm{~kg} / \mathrm{m}^{2}$ (range 19-25). All procedures were effectively completed and there were no postoperative complications such as anastomotic leakages, bleeding, ileus, wound infections, or hernias. The length of hospital stay was 9 days. In the final pathologic staging, there were two patients with stage I, two patients with stage II, and five patients with stage III. The mean tumor diameter was $4 \mathrm{~cm}$, the mean tumor-free resection margin was $11 \mathrm{~cm}$, and the mean harvested lymph nodes were 25 .

Conclusion: Single incision laparoscopic CME with CVL for advanced transverse colon cancer is safe and feasible using the pincer movement method.

Acknowledgment: We express our sincere thanks to Prof. Masaki Fukunaga for valuable comments on this article.

No competing financial interests exist.

Consent for the publication of this case report and any additional related information was taken from the patient involved in the study.

Runtime of video: 8 mins 50 secs

Keywords: complete mesocolic excision, transverse colon cancer, single incision laparoscopic surgery

\section{Cite this video}

Goutaro Katsuno, Masaki Fukunaga, Yasuhiko Nakata, Nobuyuki Kubota, Teruo Kaiga, Takao Mamiya, Masahiro Yan, Naoaki Shimamoto, Single Incision Laparoscopic Complete Mesocolic Excision with Central Vascular Ligation for Advanced Transverse Colon Cancer Using Pincer Movement Method, Videoscopy. 2017, DOI: 10.1089/vor.2017.0468.

\section{References}

1. Bucher P, Pugin F, Morel P. Single-port-access laparoscopic right hemicolectomy. Int J Colorectal Dis 2008;23:1013-1016.

2. Remzi FH, Kirat HT, Kaouk JH, Geisler DP. Single-port laparoscopy in colorectal surgery. Colorectal Dis 2008;10:823-826.

3. Romanelli JR, Earle DB. Single-port laparoscopic surgery: An overview. Surg Endosc 2009;23: 1419-1427.

4. Katsuno G, Fukunaga M, Nagakari K, Yoshikawa S, Ouchi M, Hirasaki Y. Single-incision laparoscopic colectomy for colon cancer: Early experience with 31 cases. Dis Colon Rectum 2011;54:705-710. 
5. Katsuno G, Fukunaga M, Nagakari K, Yoshikawa S, Azuma D, Hirasaki Y, Azuma D. Natural orifice specimen extraction using prolapsing technique in single-incision laparoscopic colorectal resections for colorectal cancers. Asian J Endosc Surg 2014;7:85-88.

6. Katsuno G, Fukunaga M, Nagakari K, Yoshikawa S, Azuma D, Kohama S. Short-term and long-term outcomes of single-incision versus multi-incision laparoscopic resection for colorectal cancer: A propensity-score-matched analysis of 214 cases. Surg Endosc 2016;30:1317-1325.

Original Publication Date: 2017 